\title{
Odd and even repetition sequences of independent domination number
}

\author{
Leomarich F. Casinillo \\ Department of Mathematics and Physics \\ Visayas State University \\ Visca, Baybay City, Leyte, Philippines \\ e-mail: leomarich_casinillo@yahoo.com
}

Received: 26 February 2019 Revised: 19 December 2019 Accepted: 17 January 2020

\begin{abstract}
Let $\left\{P_{n}\right\}_{n=1}^{\infty}$ be a sequence of paths. The odd repetition sequence denoted by $\left\{\rho_{k}^{o}: k \in \mathbb{N}\right\}$ is a sequence of natural numbers in which odd numbers are repeated once and defined by $\left\{\rho_{k}^{o}\right\}=\{1,1,2,3,3,4,5,5, \ldots\}=\left\{i\left(P_{n}\right)\right\}$ where $n=2 k-1$. The even repetition sequence denoted by $\left\{\rho_{k}^{e}: k \in \mathbb{N}\right\}$ is a sequence of natural numbers, in which even numbers are repeated once and defined by $\left\{\rho_{k}^{e}\right\}=\{1,2,2,3,4,4,5,6,6, \ldots\}=\left\{i\left(P_{n}\right)\right\}$, where $n=2 k$. In this paper, the explicit formula that shows the values of the element of two sequences $\left\{\rho_{k}^{o}\right\}$ and $\left\{\rho_{k}^{e}\right\}$ that depends on the subscript $k$ were constructed. Also, the formula that relates the partial sum of the elements of the said sequences, which depends on the subscript $k$ and order of the sequence of paths, were established. Further, the independent domination number of the triangular grid graph $T_{m}=\left(V\left(T_{m}\right), E\left(T_{m}\right)\right)$ will be determined using the said sequences and the two sequences will be evaluated in relation to the Fibonacci sequence $\left\{F_{n}\right\}$ along with the order of the path.
\end{abstract}

Keywords: Odd repetition sequence, Even repetition sequence, Independent domination number, Fibonacci numbers, Triangular grid graph.

2010 Mathematics Subject Classification: 05C69.

\section{Introduction}

In the theory of graphs, the idea of domination introduced by Claude Berge in 1958 [5] and followed by Oystein Ore in 1962 [9] remains intriguing and receiving much attention to mathematical research. Each domination parameter has some specified property. One important type of domination parameter is the independent domination in graphs [3, 4, 7, 10,13]. A graph $P_{n}$ is a pair of $\left(V\left(P_{n}\right), E\left(P_{n}\right)\right)$, where $V\left(P_{n}\right)$ is a finite non-empty set called the vertex-set of $P_{n}$ and $E\left(P_{n}\right)$ is a set of unordered pairs $\{u, v\}$ or simply $u v$ of distinct elements from $E\left(P_{n}\right)$ 
called the edge-set of $P_{n}$. A walk is a sequence $u_{1}, u_{2}, \ldots, u_{n}$ of vertices of graph $G$ such that $\left\{u_{i}, u_{i+1}\right\} \in E\left(P_{n}\right)$ for each $i=1,2, \ldots, n$. Vertices $u_{1}$ and $u_{n}$ are the endpoints of the walk, while the vertices $u_{2}, u_{3}, \ldots, u_{n-1}$ are internal vertices of the walk. The length of walk is the number of edges on the walk, i.e., the walk $u_{1}, u_{2}, \ldots, u_{n}$ has length $n-1$. A path is a walk that does not repeat edges and does not end where it starts, i.e., $u_{1} \rightarrow u_{2} \rightarrow \ldots \rightarrow u_{n}, u_{1} \neq u_{n}$. A path of order $n$ and length $n-1$ is denoted by $P_{n}[1,2,10]$. Then, neighborhood of $v$ is the set $N_{P_{n}}(v)=N(v)=\left\{u \in V\left(P_{n}\right): u v \in E\left(P_{n}\right)\right\}$. If $Z \subseteq V\left(P_{n}\right)$, then the open neighborhood of $Z$ is the set $N_{P_{n}}(Z)=N(Z)=\bigcup_{v \in Z} N_{P_{n}}(v)$. The closed neighborhood of $Z$ is $N_{P_{n}}[Z]=N[Z]=Z \cup N(Z)$. A subset $D$ of $V\left(P_{n}\right)$ is a dominating set of $P_{n}$ if for every $v \in V\left(P_{n}\right) \backslash D$, there exists $u \in D$ such that $u v$ is an edge of $P_{n}$, i.e., $N[D]=V\left(P_{n}\right)$. The domination number of $P_{n}$ denoted by $\gamma\left(P_{n}\right)$ is the smallest cardinality of the dominating set of $P_{n}$. For example, let $P_{4}$ be a path of order 4, then we have $\gamma\left(P_{4}\right)=2$. Let $v \in V\left(P_{n}\right)$. A dominating set $I \subseteq V\left(P_{n}\right)$ is called an independent dominating set of $P_{n}$ if no two dominating vertices in the set are adjacent. The independent domination number of $P_{n}$ denoted by $i\left(P_{n}\right)$ is the smallest cardinality of an independent dominating set of $P_{n}$. An independent dominating set of cardinality $i\left(P_{n}\right)$ is called an $i$-set. For example, let $P_{5}$ be a path of order 5 , then we have $i\left(P_{5}\right)=$ 2. The degree of vertex $v \in V\left(P_{n}\right)$, denoted by $\operatorname{deg}_{P_{n}}(v)=\operatorname{deg}(v)$, is the number of edges incident with $v$ in $P_{n}$.

In combinatorics, a multiset is a collection of objects, taken without regard to order, and with repetitions of the same object allowed. For instance, $M=\{1,1,2,2, \ldots, 9,9\}$ is a multiset. Since the order in which the objects listed in $M$ is immaterial, we could write $M$ in many other possibilities. A sequence is a set of numbers that follows a recurrence relation. We denote the first element of a sequence by $a_{1}$, the second element by $a_{2}$, the third element by $a_{3}$, and so on. The $n$-th element is $a_{n}$, called the general element of the sequence. Suppose that we have a sequence that satisfies a certain recurrence relation and initial conditions. It is often helpful to know an explicit formula for the sequence, especially if we need to compute terms with very large subscripts or if we need to examine general properties for the sequence. The odd repetition sequence denoted by $\left\{\rho_{k}^{o}: k \in \mathbb{N}\right\}$ is a sequence of natural numbers in which odd numbers are repeated once and defined by $\rho_{k}^{o}=i\left(P_{n}\right)$ where $n=2 k-1$. For instance, if $n=11$, then it implies that $\rho_{6}^{o}=i\left(P_{11}\right)=4$. The even repetition sequence denoted by $\left\{\rho_{k}^{e}: k \in \mathbb{N}\right\}$ is a sequence of natural numbers in which even numbers are repeated once and defined by $\rho_{k}^{e}=i\left(P_{n}\right)$ where $n=2 k-1$. For instance, if $n=8$, then it implies that $\rho_{4}^{o}=i\left(P_{8}\right)=3$. Figure 1 illustrates the sequence of paths of odd and even order $n$ with odd and even repetition sequence of independent domination number, respectively.
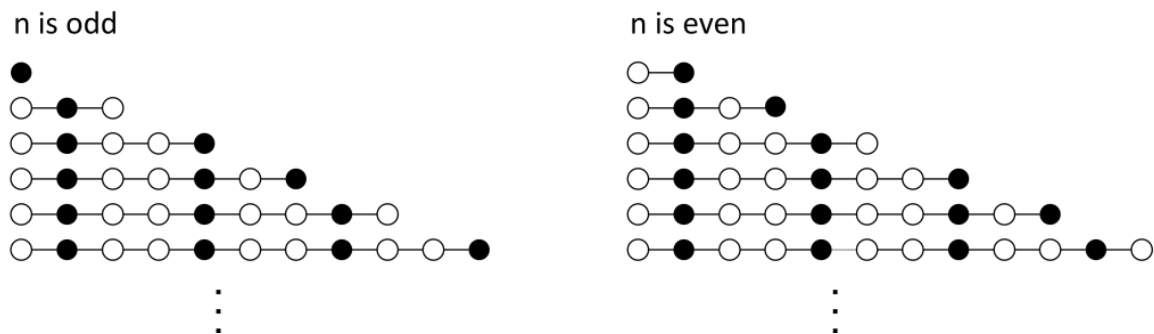

Figure 1. Sequence of paths with odd and even repetition sequence of independent domination number. 
Let $T_{m}=\left(V\left(T_{m}\right), E\left(T_{m}\right)\right)$ be a triangular grid graph where $m \in \mathbb{N}$. A graph $T_{m}$ is a subgraph of a tiling of the plane with equilateral triangles defined by the finite number of triangles called cells. The order of graph $T_{m}$ is a triangular number, that is, $\left|T_{m}\right|=\frac{m(m+1)}{2}$, where $\left|T_{m}\right|$ is the $m^{\text {th }}$ triangular number $[4,6]$. Figure 2 shows the independent dominating vertices in $T_{m}$ that follow a unique configuration of odd and even repetition sequences when $m \equiv 1(\bmod 2)$ and $m \equiv 0(\bmod 2)$, respectively.
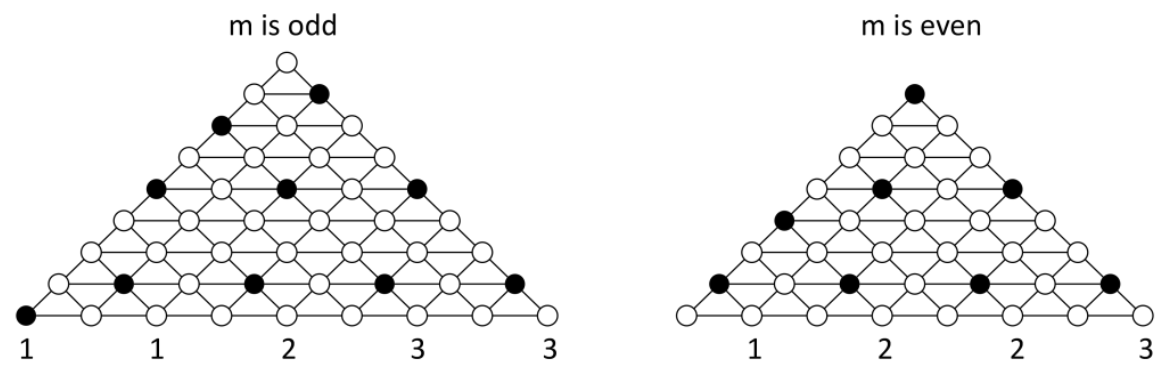

Figure 2. Independent domination number of graph $T_{m}$.

A Fibonacci number $F_{n}$ can be obtained by the following equation, $F_{n}=F_{n-2}+F_{n-1}$, for $n \in \mathbb{N} \backslash\{1,2\}$, where $F_{1}=F_{2}=1[2,12]$. The Fibonacci sequence has been widely studied by many researchers and covers a wide range of interests in combinatorial mathematics as it appears intriguing in the works of Vajda [14]. Also, the ratio of two consecutive Fibonacci numbers converges to the golden mean, that is, $\theta=\frac{1+\sqrt{5}}{2}$. This ratio has numerous applications in modern science $[8,11]$.

In this paper, we developed a formula that shows the values of the two sequences $\left\{\rho_{k}^{o}\right\}$ and $\left\{\rho_{k}^{e}\right\}$ that depends on the subscript $k$ and the formula that relates the partial sum of the elements of the said sequences which depends on the subscript $k$ and order of the sequence of paths were also constructed. We also determined the independent domination number of triangular grid graph $T_{m}$ for any positive integer $m$ in connection to the said sequences. Also, the two sequences will be evaluated in relation to Fibonacci sequence $\left\{F_{n}\right\}$ along with the order of the sequence paths and discuss some important results.

\section{Results}

The following result below is immediate from the definition of independent domination number of path with order $n \in \mathbb{N}$.

Remark 2.1. [2] Let $P_{n}$ be a path of order $n \in \mathbb{N}$. Then,

$$
i\left(P_{n}\right)= \begin{cases}\frac{n}{3} & \text { if } n \equiv 0(\bmod 3) \\ \frac{n+2}{3} & \text { if } n \equiv 1(\bmod 3) \\ \frac{n+1}{3} & \text { if } n \equiv 2(\bmod 3)\end{cases}
$$


The following Theorem is constructed from Remark 2.1. This Theorem generates the odd repetition sequence of independent domination number in the sequence of paths with odd orders.

Theorem 2.1. Let $\left\{P_{n}\right\}$ be a sequence path. If $n=2 k-1$ where $k \in \mathbb{N}$, then odd repetition sequence is obtained, that is,

$$
\left\{\rho_{k}^{o}=i\left(P_{n}\right)\right\} .
$$

Proof. To prove this theorem, we consider the following two cases below:

Case 1. If $n \equiv 0(\bmod 3)$, then it implies that for all $u \in I$ there exists $v \in V\left(P_{n}\right) \backslash I$ such that $u v \in E\left(P_{n}\right), \operatorname{deg}(u)=2$ and by Remark 2.1, clearly, $\bigcap_{j=1}^{r} N\left(u_{j}\right)=\emptyset$ where $r=\frac{n}{3}$. Since $n$ is odd, it obviously follows that there are $\frac{n}{3}$ odd dominating vertices in $P_{n}$. Therefore, we obtained an odd sequence of numbers, that is, $\{1,3,5, \ldots\}$. Now, we let $n \equiv 1(\bmod 3)$. If $n=1$, then it follows that $i\left(P_{n}\right)=1$. Hence, the first element in the sequence is 1 , that is, $\rho_{1}^{o}=1$. If $n>1$, then by Remark 2.1, there is a unique configuration that there exists $u \in I$, $\operatorname{deg}(u)=1$ such that $u v \in E\left(P_{n}\right)$ where $v \in V\left(P_{n}\right) \backslash I$ and other even domination number $\mu$, that is, $\rho_{k}^{o}=i\left(P_{n}\right)=$ $\mu+1$ such that for all $w_{j} \in I \backslash\{u\}$ and $\bigcap_{j=1}^{m} N\left(w_{j}\right)=\emptyset$, where $m=\frac{n-1}{3}$. Thus, we also obtained an odd sequence, that is, $\{1,3,5, \ldots\}$.

Case 2. Suppose that $n \equiv 2(\bmod 3)$. Obviously, we have a unique arrangement that there exists $u \in I$ such that $\operatorname{deg}(u)=1$ and there exists another odd domination number $\tau$, that is, $\rho_{k}^{o}=i\left(P_{n}\right)=\tau+1$ such that for all $w_{j} \in I \backslash\{u\}$ and $\bigcap_{j=1}^{s} N\left(w_{j}\right)=\emptyset$, where $s=\frac{n+1}{3}$ by Remark 2.1. Thus, we obtained an even sequence, that is, $\{2,4,6, \ldots\}$.

Combining the two cases, it implies that the independent domination number of the sequence of paths with odd order is an odd repetition sequence, that is, $\left\{\rho_{k}^{o}\right\}=\{1,1,2,3,3,4,5,5, \ldots\}$. This completes the proof.

By Remark 2.1 and Theorem 2.1, the following theorem is immediate. This theorem will determine the element of odd repetition sequence at any $n \in \mathbb{N}$, where the orders of the sequence of paths are odd numbers.

Theorem 2.2. Let $\left\{\rho_{k}^{o}\right\}$ be an odd repetition sequence where $k \in \mathbb{N}$ and $\left\{P_{n}\right\}$ be a sequence path where $n=2 k-1$. Then,

$$
\rho_{k}^{o}=i\left(P_{n}\right)= \begin{cases}\frac{2 k}{3} & \text { if } k \equiv 0(\bmod 3) \\ \frac{2 k+1}{3} & \text { if } k \equiv 1(\bmod 3) \\ \frac{2 k-1}{3} & \text { if } k \equiv 2(\bmod 3)\end{cases}
$$

Proof. If $n=2 k-1$, then we consider the following cases:

Case 1 . Let $k \equiv 0(\bmod 3)$. So, $k=3 a$, for some $a \in \mathbb{N}$. By Remark 2.1 and Theorem 2.1, it simply follows that there are $a=\frac{k}{3}$ odd independent domination numbers that repeat in the odd repetition sequence of $\left\{P_{n}\right\}$. Therefore, we obtained $\rho_{k}^{o}=i\left(P_{n}\right)=k-a=k-\frac{k}{3}=\frac{2 k}{3}$. 
Case 2. Let $k \equiv 1(\bmod 3)$. Obviously we have, $k=3 b+1$, for some $b \in \mathbb{N}$. In regards to Remark 2.1 and Theorem 2.1, it obviously follows that there are $b=\frac{k-1}{3}$ odd independent domination numbers that repeat in the sequence. Clearly, $\rho_{k}^{o}=i\left(P_{n}\right)=k-b=k-\frac{k-1}{3}=$ $\frac{2 k+1}{3}$.

Case 3 . Let $k \equiv 2(\bmod 3)$. Then, $k=3 c+2$, for some $b \in \mathbb{N}$. Hence, this implies that there are $c=\frac{k-2}{3}$ odd numbers that repeat in the sequence by Remark 2.1 and Theorem 2.1. Therefore, it implies that $\rho_{k}^{o}=i\left(P_{n}\right)=k-c-1=k-\frac{k-2}{3}-1=\frac{2 k-1}{3}$.

If we combine these three cases, then this completes the proof.

Next, the following Corollary is direct consequence of Theorem 2.2.

Corollary 2.1. For any $j \in \mathbb{N}, \rho_{3 j}^{o}+\rho_{3 j+3}^{o}=\rho_{3 j+1}^{o}+\rho_{3 j+2}^{o}$.

The following results, Corollary 2.2 and Corollary 2.3, will determine the parity of the element of odd repetition sequence at any subscript $k$.

Corollary 2.2. Let $n=2 k-1$. If $k \equiv 0(\bmod 3)$, then $\rho_{k}^{o} \equiv i\left(P_{n}\right) \equiv 0(\bmod 2)$.

Corollary 2.3. Let $n=2 k-1$. If $k \equiv 1(\bmod 3) \operatorname{or} k \equiv 2(\bmod 3)$, then $\rho_{k}^{o} \equiv i\left(P_{n}\right) \equiv 0(\bmod 2)$.

Using the corollaries above, we arrived at the following results that determine the order of paths in the sequence given a particular element of the odd repetition sequence.

Theorem 2.3. Let $n=2 k-1$. If $\rho_{k}^{o} \equiv i\left(P_{n}\right) \equiv 0(\bmod 2)$, then, $n=3 \rho_{k}^{o}-1$.

Proof. We suppose that $\rho_{k}^{o} \equiv i\left(P_{n}\right)$, where $n=2 k-1$. If we consider that $\rho_{k}^{o} \equiv 0(\bmod 2)$, then it follows that, $k \equiv 0(\bmod 3)$ by Corollary 2.2. By Theorem 2.2, it implies that $\rho_{k}^{o} \equiv i\left(P_{n}\right)=\frac{2 k}{3}$. Obviously, it follows that $k=\frac{3 \rho_{k}^{o}}{2}$. Thus, we end up with $n=3 \rho_{k}^{o}-1$. This completes the proof.

Theorem 2.4. Let $n=2 k-1$. If $\rho_{k}^{o} \equiv i\left(P_{n}\right) \equiv 1(\bmod 2)$, then, $n=3 \rho_{k}^{o}-2$ or $n=3 \rho_{k}^{o}$.

Proof. Supposing $\rho_{k}^{o} \equiv i\left(P_{n}\right)$, where $n=2 k-1$. Now, if we consider that $\rho_{k}^{o} \equiv 1(\bmod 2)$, then by Corollary 2.2 we have, $k \equiv \tau(\bmod 3)$, where $\tau=1,2$ and, it implies that $\rho_{k}^{o} \equiv i\left(P_{n}\right)=\frac{2 k+1}{3}$ or $\rho_{k}^{o} \equiv i\left(P_{n}\right)=\frac{2 k-1}{3}$ by Theorem 2.2. Clearly, it follows that $k=\frac{3 \rho_{k}^{o}-1}{2}$ or $k=\frac{3 \rho_{k}^{o}+1}{2}$. Therefore, $n=3 \rho_{k}^{o}-2$ or $n=3 \rho_{k}^{o}$. This completes the proof.

The next theorem is immediate from Theorem 2.2. This theorem determines the explicit formula of the partial sum of odd repetition sequence of independent domination numbers of $\left\{P_{n}\right\}$.

Theorem 2.5. Let $S_{k}^{o}=\sum_{j=1}^{k} \rho_{j}^{o}$ be a partial sum of odd repetition sequence where $k \in \mathbb{N}$ and $\left\{P_{n}\right\}$ be a sequence path where $n=2 k-1$. Then,

$$
S_{k}^{o}=\sum_{j=1}^{k} \rho_{j}^{o}=\sum_{j=1}^{k} i\left(P_{2 j-1}\right)=\left\{\begin{array}{l}
\frac{k^{2}+k}{3} \quad \text { if } k \equiv \mu(\bmod 3), \mu=0 \text { or } 2 \\
\frac{k^{2}+k+1}{3}, \quad \text { if } k \equiv 1(\bmod 3)
\end{array}\right.
$$


Proof. Suppose that $S_{k}^{o}=\sum_{j=1}^{k} \rho_{j}^{o}$ is a series of odd repetition sequence in the sequence of paths of odd orders. We consider the following cases:

Case 1 . If we consider that $k \equiv 0(\bmod 3)$, then by Theorem 2.2 we have $\frac{2 k}{3}$ consecutive natural numbers in the odd repetition sequence and $\frac{k}{3}$ consecutive odd numbers that repeat in the said sequence. It implies that $S_{k}^{o}=\sum_{j=1}^{k} \rho_{j}^{o}=\sum_{j=1}^{k} i\left(P_{2 j-1}\right)=\sum_{j=1}^{2 k / 3} j+\sum_{j=1}^{k / 3}(2 j-1)$. Simplifying the equation, we obtain $S_{k}^{o}=\sum_{j=1}^{k} \rho_{j}^{o}=\sum_{j=1}^{k} i\left(P_{2 j-1}\right)=\frac{k^{2}+k}{3}$.

Case 2. Next, if we consider $k \equiv 1(\bmod 3)$, then this implies that we have $\frac{2 k+1}{3}$ consecutive natural numbers in the odd repetition sequence and $\frac{k-1}{3}$ consecutive odd numbers that repeat in the said sequence, by Theorem 2.2. Thus, it follows that $S_{k}^{o}=\sum_{j=1}^{k} \rho_{j}^{o}=\sum_{j=1}^{k} i\left(P_{2 j-1}\right)=$ $\sum_{j=1}^{(2 k+1) / 3} j+\sum_{j=1}^{(k-1) / 3}(2 j-1)$. Hence, simplifying the equation, we end up with $S_{k}^{o}=$ $\sum_{j=1}^{k} \rho_{j}^{o}=\sum_{j=1}^{k} i\left(P_{2 j-1}\right)=\frac{3 k^{2}+k+1}{3}$.

Case 3. Lastly, we consider that $k \equiv 2(\bmod 3)$. Then, by Theorem 2.2 this implies that there are $\frac{2 k-1}{3}$ consecutive natural numbers in the odd repetition sequence and $\frac{k+1}{3}$ consecutive odd numbers that repeat in the said sequence. Hence, the series follows that $S_{k}^{o}=\sum_{j=1}^{k} \rho_{j}^{o}=$ $\sum_{j=1}^{k} i\left(P_{2 j-1}\right)=\sum_{j=1}^{2 k / 3} j+\sum_{j=1}^{k / 3}(2 j-1)$. So, by simplifying the equation, we have $S_{k}^{o}=$ $\sum_{j=1}^{k} \rho_{j}^{o}=\sum_{j=1}^{k} i\left(P_{2 j-1}\right)=\frac{3 k^{2}+k+1}{3}$.

If we combine these three cases, then this completes the proof.

The following result is immediate consequence of Theorem 2.5. This theorem determines the independent domination number of graph $T_{m}$ in relation to series of odd repetition independent domination number of the sequence of paths.

Theorem 2.6. Let $T_{m}$ be a triangular grid graph. If $m \equiv 1(\bmod 2)$, then

$$
i\left(T_{m}\right)=\left\{\begin{array}{l}
\frac{k^{2}+k}{3} \quad \text { if } k \equiv \mu(\bmod 3), \mu=0 \text { or } 2 . \\
\frac{k^{2}+k+1}{3}, \quad \text { if } k \equiv 1(\bmod 3)
\end{array}\right.
$$

where $m=2 k-1$.

Proof. Suppose that $m \equiv 1(\bmod 2)$. Then, $m=2 k-1$ implies that there are $k=\frac{m+1}{2}$ sequences of slanting paths of order $n \equiv 1(\bmod 2)$ and there are $\frac{m-1}{2}$ sequence of slanting paths of order $n \equiv 0(\bmod 2)$. By Theorem 2.1 , the total independent domination number of slanting paths of odd order is given by $\sum_{j=1}^{k} i\left(P_{2 j-1}\right)$. And since for all $u \in I$ in slanting paths of odd order, we have $\bigcup_{u \in I} N[u]=V\left(T_{m}\right)$, then it is concluded that $i\left(T_{m}\right)=\sum_{j=1}^{k} i\left(P_{2 j-1}\right)$ where $m=$ $2 k-1$. Thus, by Theorem 2.5 the proof is complete.

The next result of odd repetition sequence of independent domination number of $\left\{P_{n}\right\}$ is related to the famous Fibonacci sequence $\left\{F_{n}\right\}$. 
Theorem 2.7. If $n \equiv 1(\bmod 2)$ and $i\left(P_{n}\right)=F_{j} \equiv 0(\bmod 2)$ for some $j \in \mathbb{N}$, then $n \equiv$ $2(\bmod 3)$.

Proof. Suppose that $n \equiv 1(\bmod 2)$ and $\rho_{k}^{o} \equiv i\left(P_{n}\right)=F_{j} \equiv 0(\bmod 2)$ for some $j \in \mathbb{N}$. Then, we consider the following cases:

Case 1. If $\rho_{k}^{o} \equiv i\left(P_{n}\right)=F_{3}=2$, then clearly by Remark $2.1, n=5 \equiv 2(\bmod 3)$.

Case 2. If we consider that $\rho_{k}^{o} \equiv i\left(P_{n}\right)=F_{j} \geq 8$, then there is a unique configuration that there exists an odd number of independent dominating vertices, say $u \in I$, $\operatorname{deg}(u)=2$ and any $x y \in V\left(P_{n}\right) \backslash I$ such that $N(x) \cap N(y)=\{u\}$ and $\bigcap_{\text {all } j} N\left(u_{j}\right)=\phi$. And there exist two unique vertices in path, that is, $w \in I \backslash\left\{u_{j}\right\}, \operatorname{deg}(w)=1$ and $z \in V\left(P_{n}\right) \backslash I$ such that $N(w)=\{z\}$ and $N(z)=\{w, x\}$. Thus, it follows that there are $n-2$ vertices in $P_{n}$ such that $n-2 \equiv 0(\bmod 3)$. Clearly, this implies that $n \equiv 2(\bmod 3)$.

Combining cases (1) and (2), this completes the proof.

The following results are immediate consequences of Theorem 2.7.

Corollary 2.4. If $n \equiv 1(\bmod 2)$ and $\rho_{k}^{o} \equiv i\left(P_{n}\right)=F_{j} \equiv 0(\bmod 2)$, then $n=3 F_{j}-1$ for some $j \in \mathbb{N}$.

Corollary 2.5. If $n \equiv 1(\bmod 2)$ and $\rho_{k}^{o} \equiv i\left(P_{n}\right)=F_{j} \equiv 0(\bmod 2)$, then $\rho_{3 F_{j} / 2}^{o}=i\left(P_{n}\right)$ for some $j \in \mathbb{N}$.

Theorem 2.8. If $n \equiv 1(\bmod 2)$ and $\rho_{k}^{o} \equiv i\left(P_{n}\right)=F_{j} \equiv 1(\bmod 2)$ for some $j \in \mathbb{N}$, then $n \equiv 0(\bmod 3)$ or $n \equiv 1(\bmod 3)$.

Proof. If we suppose that $n \equiv 1(\bmod 2)$ and $\rho_{k}^{o} \equiv i\left(P_{n}\right)=F_{j} \equiv 1(\bmod 2)$ for some $j \in \mathbb{N}$, then we consider the following cases:

Case 1 . If $\rho_{k}^{o} \equiv i\left(P_{n}\right)=F_{2} \equiv 1$, then by Remark 2.1 , we obtained $n=3 \equiv 0(\bmod 3)$ or $n=1 \equiv 1(\bmod 3)$.

Case 2. Suppose that $\rho_{k}^{o} \equiv i\left(P_{n}\right)=F_{j} \geq 3$. Then, there exists an even number of independent dominating vertices, say $u \in I, \operatorname{deg}(u)=2$ and $\bigcap_{\text {all } j} N\left(u_{j}\right)=\phi$ and there exists a unique independent dominating vertex $w \in I \backslash\left\{u_{j}\right\}, \operatorname{deg}(w)=1$ and non-dominating vertex $x \in V\left(P_{n}\right) \backslash I$, such that $x \in N(w) \cap N(u)$. Hence, it follows that $n \equiv 1(\bmod 3)$. Further, there exists also a particular configuration that if for all $v \in I, \operatorname{deg}(v)=2$ and $\bigcap_{\text {all } j} N\left(v_{j}\right)=\phi$, then it is clear that $n \equiv 0(\bmod 3)$.

Therefore, if we combine the two cases, then this completes the proof.

The next Corollaries are quick consequences of Theorem 2.8 .

Corollary 2.6. If $n \equiv 1(\bmod 2)$ and $\rho_{k}^{o} \equiv i\left(P_{n}\right)=F_{j} \equiv 1(\bmod 2)$, then $n=3 F_{j}-2$ orn $=$ $3 F_{j}$ for some $j \in \mathbb{N}$.

Corollary 2.7. If $n \equiv 1(\bmod 2)$ and $\rho_{k}^{o} \equiv i\left(P_{n}\right)=F_{j} \equiv 1(\bmod 2)$, then $\rho_{\left(3 F_{j}-1\right) / 2}^{o}=i\left(P_{n}\right)$ or $\rho_{\left(3 F_{j}+1\right) / 2}^{o}=i\left(P_{n}\right)$ for some $j \in \mathbb{N}$. 
Again by Remark 2.1, the following theorem is constructed. This theorem generates the even repetition sequence of independent domination number in the sequence of paths with even orders.

Theorem 2.9. Let $\left\{P_{n}\right\}$ be a sequence path. If $n=2 k, k \in \mathbb{N}$, then an even repetition sequence is obtained, that is

$$
\left\{\rho_{k}^{e}=i\left(P_{n}\right)\right\}
$$

Proof. If we suppose that $n \equiv 0(\bmod 2)$, then we consider the following cases:

Case 1 . Let $n \equiv 2(\bmod 3)$. If $n=2$, then it is easy to check that $\rho_{1}^{e}=i\left(P_{2}\right)=1$. If $n>2$, then there is an arrangement of independent dominating set that there exists $u \in I, \operatorname{deg}(u)=1$ such that $u v \in E\left(P_{n}\right)$ where $v \in V\left(P_{n}\right) \backslash I$ and there exists also another even domination number $x$, that is, $\rho_{k}^{e}=i\left(P_{n}\right)=x+1$ such that for all $w_{j} \in I \backslash\{u\}, \cap \quad{ }_{j=1}^{r} N\left(w_{j}\right)=\{\}$, where $r=\frac{n+1}{3}$ by Remark 2.1. Thus, we obtained an odd sequence, that is, $\{1,3,5, \ldots\}$.

Case 2. Let $n \equiv 1(\bmod 3)$. If $n=4$, then it is clear that $\rho_{2}^{e}=i\left(P_{4}\right)=2$. Now, if $n>4$, then it implies that there exists $u, v \in I, \operatorname{deg}(u)=1, \operatorname{deg}(v)=2$ such that $N(u) \cap N(v)=\{w\}$, where $w \in V\left(P_{n}\right) \backslash I$. And by Remark 2.1, there exists another even independent domination number, say $y$, that is $\rho_{k}^{e}=i\left(P_{n}\right)=y+2$ such that for all $w \in I \backslash\{u, v\}, \cap_{j=1}^{s} N\left(w_{j}\right)=\emptyset$, where $r=\frac{n-4}{3}$. Thus, this implies that we obtained an even sequence, that is, $\{2,4,6, \ldots\}$.

Case 3. Let $n \equiv 0(\bmod 3)$. Since $n$ is even, let $n=2 a$ for all positive integer $a$. Also, since $n$ is divisible by 3 , then it can be written as $2 a \equiv 0(\bmod 3)$, which implies that $2 a=3 b$ for some positive integer $b$. It means that for all $u \in I, \cap_{j=1}^{t} N\left(u_{j}\right)=\emptyset$ where $t=\frac{n}{3}$ by Remark 2.1. Obviously, we have $b=2\left(\frac{a}{3}\right)$ and it follows that we have an even integer sequence of independent domination number, that is,$\{2,4,6, \ldots\}$.

Thus, combining all cases, it implies that the independent domination number of the sequence of paths with even order is an even repetition sequence, that is,

$$
\left\{\rho_{k}^{e}\right\}=\{1,2,2,3,4,4,5,6,6, \ldots\}
$$

This completes the proof.

The following theorem is a quick consequence of Remark 2.1 and Theorem 2.9. This theorem will determine the element of even repetition sequence at any subscript $k \in \mathbb{N}$.

Theorem 2.10. Let $\left\{\rho_{k}^{e}\right\}$ be an even repetition sequence where $k \in \mathbb{N}$ and $\left\{P_{n}\right\}$ be a sequence path where $n=2 k$. Then,

$$
\rho_{k}^{e}=i\left(P_{n}\right)= \begin{cases}\frac{2 k}{3} & \text { if } k \equiv 0(\bmod 3) \\ \frac{2 k+1}{3} & \text { if } k \equiv 1(\bmod 3) \\ \frac{2 k+2}{3} & \text { if } k \equiv 2(\bmod 3)\end{cases}
$$

Proof. Suppose that $n=2 k$. We consider the following cases: 
Case 1. Let $k \equiv 0(\bmod 3)$. Clearly, it follows that $k=3 x$, for some $x \in \mathbb{N}$. It implies that there are $x=\frac{k}{3}$ even independent domination numbers that repeat in the even repetition sequence by Remark 2.1 and Theorem 2.9. So, we obtained $\rho_{k}^{e}=i\left(P_{n}\right)=k-x=k-\frac{k}{3}=\frac{2 k}{3}$.

Case 2 . Let $k \equiv 1(\bmod 3)$. Then, $k=3 y+1$, for some $y \in \mathbb{N}$. And by Remark 2.1 and Theorem 2.9, it follows that there are $x=\frac{k-1}{3}$ even independent domination numbers that repeat in the sequence. So, it implies that we have $\rho_{k}^{e}=i\left(P_{n}\right)=k-y=k-\frac{k-1}{3}=\frac{2 k+1}{3}$.

Case 3 . Let $k \equiv 2(\bmod 3)$. Then, $k=3 z+2$, for some $z \in \mathbb{N}$. By Remark 2.1 and Theorem 2.9, this implies that there are $z=\frac{k-2}{3}$ even independent domination numbers that repeat in the sequence. It follows directly that $\rho_{k}^{e}=i\left(P_{n}\right)=k-z=k-\frac{k-2}{3}=\frac{2 k+2}{3}$.

Hence, if we combine all three cases, then this completes the proof.

The next Corollaries are direct consequences of Theorem 2.10.

Corollary 2.8. For any $h \in \mathbb{N}, \rho_{3 h}^{e}+\rho_{3 h+3}^{e}=\rho_{3 h+1}^{e}+\rho_{3 h+2}^{e}$

The following Corollaries 2.9 and 2.10 will determine the parity of the element of even repetition sequence at any subscript $k$.

Corollary 2.9. Let $n=2 k$. If $k \equiv 1(\bmod 3)$, then $\rho_{k}^{e} \equiv i\left(P_{n}\right) \equiv 1(\bmod 2)$.

Corollary 2.10. Let $n=2 k$. If $k \equiv 0(\bmod 3)$ or $k \equiv 2(\bmod 3)$, then $\rho_{k}^{e} \equiv i\left(P_{n}\right) \equiv 0(\bmod 2)$.

By the corollaries above, namely Corollary 2.9 and Corollary 2.10, and Theorem 2.10, we obtain the following results that determines the order of paths in the sequence given a particular element of the even repetition sequence.

Theorem 2.11. Let $n=2 k$. If $\rho_{k}^{e} \equiv i\left(P_{n}\right) \equiv 1(\bmod 2)$, then $n=3 \rho_{k}^{e}-1$.

Proof. Supposing $\rho_{k}^{e} \equiv i\left(P_{n}\right)$, where $n=2 k-1$. If we consider that $\rho_{k}^{e} \equiv 1(\bmod 2)$, then it follows from Corollary 2.9 that $k \equiv 1(\bmod 3)$ and by Theorem 2.10 , it simply implies that $\rho_{k}^{e} \equiv i\left(P_{n}\right)=\frac{2 k+1}{3}$. And it follows that $k=\frac{3 \rho_{k}^{e}-1}{2}$. Thus, we obtained $n=3 \rho_{k}^{e}-1$. This completes the proof.

Theorem 2.12. Let $n=2 k$. If $\rho_{k}^{e} \equiv i\left(P_{n}\right) \equiv 0(\bmod 2)$, then $n=3 \rho_{k}^{e}$ or $n=3 \rho_{k}^{e}-2$. Proof. We suppose that $\rho_{k}^{e} \equiv i\left(P_{n}\right)$, where $n=2 k$. And if we consider that $\rho_{k}^{e} \equiv 0(\bmod 2)$, then by Corollary 2.10 , it implies that $k \equiv \mu(\bmod 3)$, where $\mu=1,2$ and by Theorem 2.10 we obtained $\rho_{k}^{e} \equiv i\left(P_{n}\right)=\frac{2 k}{3}$ or $\rho_{k}^{e} \equiv i\left(P_{n}\right)=\frac{2 k+1}{3}$. Hence, it follows that $k=\frac{3 \rho_{k}^{e}}{2}$ or $k=\frac{3 \rho_{k}^{e}-2}{2}$. Obviously, we end up with $n=3 \rho_{k}^{e}$ or $n=3 \rho_{k}^{e}-2$. This completes the proof.

The following theorem is an immediate consequence of Theorem 2.10. The theorem gives the explicit formula for the partial sum of even repetition sequence of independent domination of the sequence of paths with even orders. 
Theorem 2.13. Let $S_{k}^{e}=\sum_{j=1}^{k} \rho_{j}^{e}$ be a partial sum of odd repetition sequences where $k \in \mathbb{N}$ and $\left\{P_{n}\right\}$ be a sequence path where $n=2 k$. Then,

$$
S_{k}^{e}=\sum_{j=1}^{k} \rho_{j}^{e}=\sum_{j=1}^{k} i\left(P_{2 j}\right)=\left\{\begin{array}{l}
\frac{k^{2}+2 k}{3} \quad \text { if } k \equiv \theta(\bmod 3), \quad \theta=0 \text { or } 1 . \\
\frac{k^{2}+2 k+1}{3}, \quad \text { if } k \equiv 2(\bmod 3)
\end{array}\right.
$$

Proof. Let $S_{k}^{e}=\sum_{j=1}^{k} \rho_{j}^{e}$ be a series of even repetition sequence in the sequence of paths of even orders. Then, we consider the following cases:

Case 1 . If we consider that $k \equiv 0(\bmod 3)$, then by Theorem 2.10 we have $\frac{2 k}{3}$ consecutive natural numbers in the even repetition sequence and $\frac{k}{3}$ consecutive even numbers that repeat in the said sequence. This implies that $S_{k}^{e}=\sum_{j=1}^{k} \rho_{j}^{e}=\sum_{j=1}^{k} i\left(P_{2 j-1}\right)=\sum_{j=1}^{2 k / 3} j+\sum_{j=1}^{k / 3}(2 j)$. Hence, simplifying the right-hand side of the equation, clearly we have

$$
S_{k}^{o}=\sum_{j=1}^{k} \rho_{j}^{o}=\sum_{j=1}^{k} i\left(P_{2 j-1}\right)=\frac{k^{2}+2 k}{3} .
$$

Case 2. Next, we suppose that $k \equiv 1(\bmod 3)$. Then, it follows that we have $\frac{2 k+1}{3}$ consecutive natural numbers in the even repetition sequence and $\frac{k-1}{3}$ consecutive even numbers that repeat in the said sequence by Theorem 2.10. This implies that we have the following equation $S_{k}^{e}=\sum_{j=1}^{k} \rho_{j}^{e}=\sum_{j=1}^{k} i\left(P_{2 j}\right)=\sum_{j=1}^{(2 k+1) / 3} j+\sum_{j=1}^{(k-1) / 3}(2 j)$.Therefore, simplifying the equation, we end up with $S_{k}^{e}=\sum_{j=1}^{k} \rho_{j}^{e}=\sum_{j=1}^{k} i\left(P_{2 j}\right)=\frac{3 k^{2}+2 k}{3}$.

Case 3 . Lastly, we suppose that $k \equiv 2(\bmod 3)$. Then, by Theorem 2.10 this implies that there are $\frac{2 k+1}{3}$ consecutive natural numbers in the even repetition sequence and $\frac{k-2}{3}$ consecutive even numbers that repeats in the said sequence. Hence, from the series follows that $S_{k}^{e}=\sum_{j=1}^{k} \rho_{j}^{e}=\sum_{j=1}^{k} i\left(P_{2 j}\right)=\sum_{j=1}^{(2 k+2) / 3} j+\sum_{j=1}^{(k-2) / 3}(2 j)$. Clearly, by simplifying the equation, we have $S_{k}^{e}=\sum_{j=1}^{k} \rho_{j}^{e}=\sum_{j=1}^{k} i\left(P_{2 j}\right)=\frac{3 k^{2}+2 k+1}{3}$.

Therefore, if we combine all three cases, then this completes the proof.

The next theorem is an immediate consequence of Theorem 2.13. This theorem determines the independent domination number of graph $T_{m}$ in relation to series of even repetition independent domination numbers of the sequence of paths.

Theorem 2.14. Let $T_{m}$ be a triangular grid graph. If $m \equiv 0(\bmod 2)$, then

$$
i\left(T_{m}\right)=\left\{\begin{array}{l}
\frac{k^{2}+2 k}{3}, \quad \text { if } k \equiv \theta(\bmod 3), \theta=0 \text { or } 1 . \\
\frac{k^{2}+2 k+1}{3}, \quad \text { if } k \equiv 2(\bmod 3)
\end{array}\right.
$$

where $m=2 k$. 
Proof. If we consider that $m \equiv 0(\bmod 2)$, then we have $m=2 k$, which implies that there are $k=\frac{m}{2}$ sequence of slanting paths of order $n \equiv 0(\bmod 2)$ and $n \equiv 1(\bmod 2)$. By Theorem 2.9, the total independent domination number of slanting paths of odd order is given by $\sum_{j=1}^{k} i\left(P_{2 j}\right)$. Since for all $v \in I$ in slanting paths of odd order, $\bigcup_{v \in I} N[v]=V\left(T_{m}\right)$, then it follows that $i\left(T_{m}\right)=\sum_{j=1}^{k} i\left(P_{2 j}\right)$, where $m=2 k$. Thus, by Theorem 2.13 the proof is complete.

The following results of even repetition sequence of independent domination number of the sequence of paths with even orders are relate to the intriguing Fibonacci sequence $\left\{F_{n}\right\}$.

Theorem 2.15. If $n \equiv 0(\bmod 2)$ and $\rho_{k}^{e}=i\left(P_{n}\right)=F_{j} \equiv 0(\bmod 2)$ for some $j \in \mathbb{N}$, then $n \equiv 1(\bmod 3)$ or $n \equiv 0(\bmod 3)$.

Proof. Let $n \equiv 0(\bmod 2)$ and $\rho_{k}^{e} \equiv i\left(P_{n}\right)=F_{j} \equiv 0(\bmod 2)$ for some $j \in \mathbb{N}$. Then, consider the following two cases:

Case 1. If $\rho_{k}^{e} \equiv i\left(P_{n}\right)=F_{3}=2$, then, clearly by Remark $2.1, n=4 \equiv 1(\bmod 3)$ or $n=6 \equiv 0(\bmod 3)$.

Case 2. Suppose that $\rho_{k}^{e} \equiv i\left(P_{n}\right)=F_{j} \geq 8$. Then, there is a particular configuration that there exists a dominating vertex, say $u \in I, \operatorname{deg}(u)=1$ such that $N(u) \cap N(v)=\{x\}$, where $v \in I \backslash\{u\}$ and $x \in V\left(P_{n}\right) \backslash I$ and $\bigcap_{\text {all } j} N\left(u_{j}\right)=\phi$. And there exists another odd independent domination number such that for all $v_{j} \in I \backslash\{u\}, \operatorname{deg}\left(v_{j}\right)=2$ and $\cap N\left(v_{j}\right)=\{\}$. Hence, it implies that $n \equiv 1(\bmod 3)$. Further, there is another arrangement that for all $u_{j} \in I$, $\operatorname{deg}\left(u_{j}\right)=2$ and $\cap N\left(u_{j}\right)$ is empty. Hence, it clearly follows that $n \equiv 1(\bmod 3)$.

Thus, combining the two cases, then this completes the proof.

The next corollaries are immediate consequences of Theorem 2.15.

Corollary 2.11. If $n \equiv 0(\bmod 2)$ and $\rho_{k}^{e} \equiv i\left(P_{n}\right)=F_{j} \equiv 0(\bmod 2)$, then $n=3 F_{j}-2$ or $n=3 F_{j}$ for some $j \in \mathbb{N}$.

Corollary 2.12. If $n \equiv 0(\bmod 2)$ and $\rho_{k}^{e} \equiv i\left(P_{n}\right)=F_{j} \equiv 0(\bmod 2)$, then $\rho_{\left(3 F_{j}-2\right) / 2}^{o}=i\left(P_{n}\right)$ or $\rho_{3 F_{j} / 2}^{o}=i\left(P_{n}\right)$ for some $j \in \mathbb{N}$.

Theorem 2.16. If $n \equiv 0(\bmod 2)$ and $\rho_{k}^{e} \equiv i\left(P_{n}\right)=F_{j} \equiv 1(\bmod 2)$ for some $j \in \mathbb{N}$, then $n \equiv 2(\bmod 3)$.

Proof. Supposing that $n \equiv 0(\bmod 2)$ and $\rho_{k}^{e} \equiv i\left(P_{n}\right)=F_{j} \equiv 1(\bmod 2)$ for some $j \in \mathbb{N}$. Then, consider the following two cases:

Case 1. If we consider that $\rho_{k}^{e} \equiv i\left(P_{n}\right)=F_{1}=F_{2} \equiv 1$, then by Remark 2.1, it simply means that $n=2 \equiv 2(\bmod 3)$.

Case 2. Now, if we suppose that $\rho_{k}^{e} \equiv i\left(P_{n}\right)=F_{j} \geq 3$, then there is a unique configuration that there exists a vertex $u \in I$ and $\operatorname{deg}(u)=2$ such that $N(x)=\{x\}$ where $x \in V\left(P_{n}\right) \backslash I$. Also, there exists another odd number of dominating vertices $v_{j} \in I \backslash\{u\}$ and $\operatorname{deg}\left(v_{j}\right)=2$ such that $\bigcap_{\text {all } j} N\left(v_{j}\right)=\phi$ and $\bigcup_{\text {all } j} N\left(v_{j}\right)=\left(V\left(P_{n}\right) \backslash I\right) \cup\{x\}$. Hence, it implies that $n \equiv 2(\bmod 3)$.

Thus, combining cases (1) and (2), this completes the proof. 
The following corollaries are consequences of Theorem 2.16.

Corollary 2.13. If $n \equiv 0(\bmod 2)$ and $\rho_{k}^{e} \equiv i\left(P_{n}\right)=F_{j} \equiv 1(\bmod 2)$, then $n=3 F_{j}-1$ for some $j \in \mathbb{N}$.

Corollary 2.14. If $n \equiv 0(\bmod 2)$ and $\rho_{k}^{e} \equiv i\left(P_{n}\right)=F_{j} \equiv 1(\bmod 2)$, then $\rho_{\left(3 F_{j}-1\right) / 2}^{e}=i\left(P_{n}\right)$ for some $j \in \mathbb{N}$.

\section{Acknowledgements}

I would like to acknowledge Emily L. Casinillo of Department of Mathematics and Physics (VSU) for the support and encouragement to finish this paper and the referees for the rigorous review and comments to improve this paper.

\section{References}

[1] Canoy, Jr. S. R., \& Garces, I. J. L. (2002). Convex sets under some graph operations. Graphs and Combinatorics, 18, 787-793.

[2] Casinillo, L. F. (2018). A note on Fibonacci and Lucas number of domination in path. Electronic Journal of Graph Theory and Applications, 6(2), 317-325.

[3] Casinillo, L. F., Lagumbay, E. T. \& Abad, H. R. F. (2017). A note on connected interior domination in join and corona of two graphs. IOSR Journal of Mathematics, 13(2), 66-69.

[4] Chartrand, G., \& Zhang, P. (2012). A First Course in Graph Theory. Dover Publication Inc., New York.

[5] Cockayne, E. J., \& Hedetniemi, S. T. (1977). Towards a theory of domination in graph. Networks Advanced Topics, 7, 247-261.

[6] Dorfling, M., \& Henning, M. A. (2006). A note on power domination in grid graphs. Discrete Applied Mathematics, 154, 1023-1027.

[7] Haynes, T. W., Hedetniemi, S. T., \& Slater, P. J. (1998). Fundamentals of Domination in Graphs. Marcel Dekker. New York.

[8] Koshy, T. (2001). Fibonacci and Lucas Numbers with Application. Wiley-Interscience, New York.

[9] Ore, O. (1962). Theory of Graphs. American Mathematical Society Provedence, R. I.

[10] Perderson, A. S., \& Vestergaard, P. D. (2005). The number of independent sets in unicyclic graphs. Discrete Applied Mathematics, 152, 246-256.

[11] Prodinger, H., \& Tichy, R. (1982). Fibonacci numbers of graphs. Fibonacci Quarterly, $20(1), 16-21$. 
[12] Singh, B. Sisodiya, K., \& Ahmad, F. (2014). On the products of $k$-Fibonacci numbers and $k$-Lucas numbers. International Journal of Mathematics and Mathematical Science, 21, $1-4$.

[13] Tarr, J. M. (2010). Domination in Graphs. Graduate Theses and Dissertations. Retrieved from https://scholarcommons.usf.edu/etd/1786.

[14] Vajda, S. (2008). Fibonacci and Lucas Numbers and the Golden Section: Theory and Applications. Dover Publications Inc., New York. 